\title{
A GPS/DR Data Fusion Method Based on the GPS Characteristics for Mobile Robot Navigation
}

\author{
Yuanliang Zhang ${ }^{1}$ and Kil To Chong ${ }^{2 *}$ \\ ${ }^{1}$ School of Mechanical Engineering, Huaihai Institute of Technology \\ ${ }^{2}$ School of Electronics and Information, Chonbuk National University \\ ${ }^{1}$ newlion79@gmail.com, ${ }^{2}$ kitchong@chonbuk.ac.kr
}

\begin{abstract}
In this paper we have considered the problem of outdoor mobile robot navigation using dead reckoning (DR) system and single GPS receiver. DR is a very simple and practical positioning technique. It is used in various positioning and navigation applications, especially for the mobile robot. DR can provide short term precise navigation information. But its errors will generally accumulate as the mobile robot continues to travel, and the calculated position of the mobile robot will become less and less accurate. For outdoor navigation application, GPS exhibit lots of advantages. It can provide real time and relatively accurate position data in spite of bad weather or other negative factors. But the big errors of civilian used single GPS receiver prevent it from applying to navigation for mobile robot alone. Differential GPS (DGPS) can be used to achieve an error of less than one meter but the costs are prohibitive in terms of commercializing it into the mass market. In this study, a cheap single GPS receiver cooperated with a DR system was used for the navigation system of a outdoors mobile robot in which a new GPS/DR data fusion method was utilized. This proposed fusion algorithm was based on the characteristics of the chosen single GPS receiver. The presented fusion algorithm does not bring much calculation burden and can provide accurate and robust navigation information for the mobile robot by adaptively switching between GPS/DR and DR when GPS lost the satellite signals. Simulation and experiment were performed to validate the effectiveness of the proposed fusion method and the good results showed its potential for outdoors mobile robot navigation.
\end{abstract}

Keywords: GPS; dead reckoning; data fusion method; mobile robot; navigation

\section{Introduction}

GPS is now widely used for outdoors navigation and has many applications such as positioning, locating, navigating, mapping, surveying and time determination. For outdoors navigation, GPS provides three dimensions position and velocity information. Under favorable conditions, GPS can provide continuous and relatively accurate navigation information over a long period of time. Much work has been carried out on the use of GPS-based navigation systems in mobile robots and autonomous vehicles [1, 2]. Seo et al., [3] described a precise outdoor localization algorithm with the integration of GPS and inertial navigation system (INS) for outdoor mobile robot. In [4] a navigation system using low accuracy inertial sensors and GPS were presented for mobile robot navigation. In this application the accuracy of the low accuracy INS is improved by the proper damping of INS errors. Adusumilli et al., [5] have presented a

\footnotetext{
* Corresponding Author
} 
random forest regression based INS and GPS integration algorithm to provide continuous and accurate navigation information. In the case of GPS outages, the introduced model could provide an INS error estimates, so that the continuity of the navigation system could be maintained.

But the errors in a civilian used single GPS receiver is still large even though the SA policy has been removed. Based on the experiments the single GPS receiver may has the errors more than 20 meters [6]. The big errors make the single GPS receiver unfit for mobile robot navigation. And when the mobile robot passes through urban areas or through forest, the positioning accuracy degrades due to the blockage of satellite's signals. As a result, the navigation solution becomes futile. In this case GPS also cannot be used continuously for mobile robot navigation due to the loss of satellite signals. Reflected signals and relatively poor geometries make GPS further less accurate [7]. Based on the experiments, we found that when the satellites detected by the GPS receiver change the accuracy of GPS will also degrade. All of these factors show that single GPS receiver cannot be used to navigate the mobile robot without any other auxiliary sensors. Differential GPS (DGPS) can provide a more precise navigation information with the error of less than one meter. But the cost of a DGPS is prohibitive and there is a need for a base station to provide the difference data. Due to these reasons fusion of GPS with other navigation sensors is necessary.

DR is one of the common techniques utilized for mobile robot positioning and navigation. The method uses mainly the wheel/motor (encoder) and heading (compass) sensors to acquire the position and path data for the mobile robot. DR system has high resolution, fast response time, and low noise. However, it also suffer from unpredictable systematic errors [8]. The analysis of DR systematic errors can be found in [9]. This system provides short-term and very precise navigation information but since the errors accumulate over time without limit, it cannot be used all by itself to navigate the mobile robot without accurate validation. On the other hand, GPS has low resolution, slow response time, and large noise, but it does not suffer from drift errors because it does not use an integration of incremental signals. In order to take advantages of sensor fusion of GPS and DR system, many algorithms are developed. Kalman filters are widely used for the sensor fusion [10, 11]. Chen et al. [12] proposed an algorithm to improve positioning precision of the GPS/INS integrated navigation system during GPS outages. This algorithm combined a strong tracking Kalman filter with wavelet neural network for INS errors compensation. Zhang and Zhao [13] introduced three nonlinear filtering algorithms, first-order extended Kalman filter (FEKF), the second-order extended Kalman filter EKF (SEKF) and EKF-Rauch-TungStriebel (EKF-RTS) smoother, based on GPS and DR. Based on the simulations, they compared these three algorithms. Numerical results demonstrate that the EKF-RTS gave clearly better estimates than the FEKF and SEKF.

Kalman filter is a good tool. But Kalman filter based approaches possess several inadequacies related to sensor error model, immunity to noise, and computational load. In details, Kalman filter need that the noise is white Gaussian noise. But the noise of GPS is complicated which cannot be white noise. This make the precision of Kalman filter based approaches decrease and sometime can make the system unstable. And the computation burden of these algorithm is also a important problem. So it is better to design a data fusion algorithm based on the characteristics of GPS data. In this paper, a cheap single GPS receiver was used to provide positioning information. Based on the experimental data collected under the good condition the GPS receiver has an error of more than eight meters. A new data fusion method was developed and it was based on 
the characteristics of the GPS receiver, and its aim is to fuse the navigation data of GPS and DR. The proposed data fusion method can provide accurate navigation data by modifying the reliability of GPS and DR which is used to decide the weights of the GPS and DR systems for providing the navigation information. And when GPS is outages the proposed algorithm can adaptively switch between GPS and GPS/DR. The simulation and experiment results show the good potential of the use of this method in mobile robot navigation.

The details of the study are broken down into several sections. Section 2 discusses information about the GPS navigation system and the characteristics of the GPS receiver used. Section 3 describes the proposed data fusion method. Section 4 details the simulation and experiment conducted whilst the conclusion is presented in Section 5.

\section{The Characteristics of the Chosen Single GPS Receiver}

GPS is a kind of global positioning system which can provide user position and velocity information by means of a hand-held portable receiver. GPS was initially developed for military use but is now widely used for civilian applications including timing, mapping, navigation, etc. It consists of three main parts: the space segment, including all GPS navigation satellites; the user segment, including all of the GPS receivers; the control segment, including monitoring systems in land stations and control stations. There are at least twenty-four operational GPS satellites at any given time and each of them continuously transmits data indicating its location and current time. The GPS satellites synchronize their transmissions so that their signals are sent at the same time and when a GPS device receives the transmission from two or more satellites, their arrival time differences is used to calculate the relative distance of the receiver to each satellite. A GPS receiver uses the pseudoranges obtained from the signals to calculate the positioning information [14]. The observation of at least four satellites simultaneously will permit determination of the 3D coordinates of the receiver and the time offset between the receiver and GPS time. It provides a practical and affordable means of determining position, velocity, and time around the globe.

The accuracy of the GPS information relies in the knowledge of the satellites' orbit and a very accurate timing mechanism. In GPS applications, the main goal is to acquire the location information position of the receiver as accurately as possible, based on its distance from the satellites. Since GPS is generally utilized outdoors, many disturbances can affect its precision. These disturbances include clock offset, satellite orbital errors, atmospheric/ ionospheric effect, multi-path effect and receiver noise. The geometric location of the receiver with respect to the satellites can also influence the accuracy of the GPS. For the two-dimensional positioning case, horizontal dilution of precision (HDOP) is used to indicate the effect of the geometry of the satellites on the precision of the GPS. The estimated position of the receiver is more accurate when the satellites recognized are geometrically well-distributed and is indicated by a small HDOP factor. Moreover, the changing location and number of available satellites over time influence the precision. Based on the experiment results when the satellites which the GPS receiver detected change, the precision of GPS will degrade. Because of so many disturbance sources, even though the SA policy was canceled, the positioning errors of single GPS receiver is still big. And since the existence of so many disturbance sources, it is very difficult to obtain the model of GPS errors.

The errors of the single GPS receiver can be more than 20 meters. It is impossible to use only single GPS to navigate the mobile robots. DGPS can provide significant improvement in accuracy over single GPS [15]. There are several ways to operate in 
differential mode, utilizing the information sent from a DGPS base station. DGPS can provide a very precise positioning service but it is expensive and requires a base station. When the DGPS receiver is far away from the base station the precision of DGPS will degrade. And in the case of losing the signal from the base station the precision of DGPS will decrease to single GPS level. So combine GPS with other navigation system is the best way to solve navigation problems. About navigation data fusion, Kalman filter based algorithms are frequently used. Kalman filter is a set of mathematical equations that provides an efficient computational (recursive) means to estimate the state of a process, in a way that minimizes the mean of the squared error. The filter is very powerful in several aspects: it supports estimations of past, present, and even future states, and it can do so even when the precise nature of the modeled system is unknown. But Kalman filter based approaches possess several inadequacies related to sensor error model, immunity to noise, and computational load. In details, Kalman filter needs that the noise is white Gaussian noise. But the noise of GPS is complicated which cannot be white noise. This make the precision of Kalman filter based approaches decrease and sometime can make the system unstable. And the computation burden of these algorithm is also an important problem.

In this paper, we utilized a cheap single GPS receiver cooperating with DR to provide the positioning information. The designed data fusion algorithm is based on the characteristics of the GPS data. In order to analyze the characteristics of the GPS data, experiments are done. We chose several typical positions, including open fields, positions in bush and positions near the high trees and buildings. In these measuring points, we did the GPS experiments in various time including early in the morning, morning, noon, afternoon, evening and night. We found that in different time since the number of satellites detected by GPS receiver is different and the atmospheric/ionospheric effect are different, the precisions of GPS are also different. The results measured in the position near high trees and buildings are worse than that in open fields due to the multi-path effect. Sometimes the satellite signals were lost. In this case the errors of GPS were very big, and GPS cannot provide positioning information. And when the satellites in the sky change, the precision of GPS will degrade. In general, the absolute errors of the selected single GPS receiver are about 8 15 meters. Despite of all of these differences, the experiment results proved one useful information which can be used to analyze the characteristics of the GPS data. All of the data collected in different positions and time show one similar feature. The details are given in the following part of this paper.

A set of typical GPS data shown in Fig. 1 is used here to do the explain. In order to make it easy to do the analysis we process the GPS data by simply subtracting the data from their mean value. The processed data is shown in Fig. 2. Fig. 2 shows the information that makes some sense. From Fig. 2 it can be seen that the successive errors of the chosen GPS receiver are tightly related, and therefore the error of GPS is strongly colored. Consider the relative position case, we subtract the current data from it last sampling time data. Fig. 3 shows the difference between the adjacent sampling time GPS data. From Figs. 2 and 3 we can see that in a short term this chosen single GPS receiver's data does not change a lot if the satellites recognized by the GPS receiver do not change and the signal from these satellites received by the GPS receiver is strong enough. And every time when the data changes the data always skip one resolution of the GPS receiver, for latitude $0.1854 \mathrm{~m}$ and for longitude $0.1503 \mathrm{~m}$. Now we can get some useful characteristics of the chosen single GPS receiver. In spite of big errors, the successive errors of the chosen GPS receiver are tightly related. In normal 
situation, the difference between the two successive data is no more than $0.1854 \mathrm{~m}$ in latitude direction, and is no more than $0.1503 \mathrm{~m}$ in longitude direction.
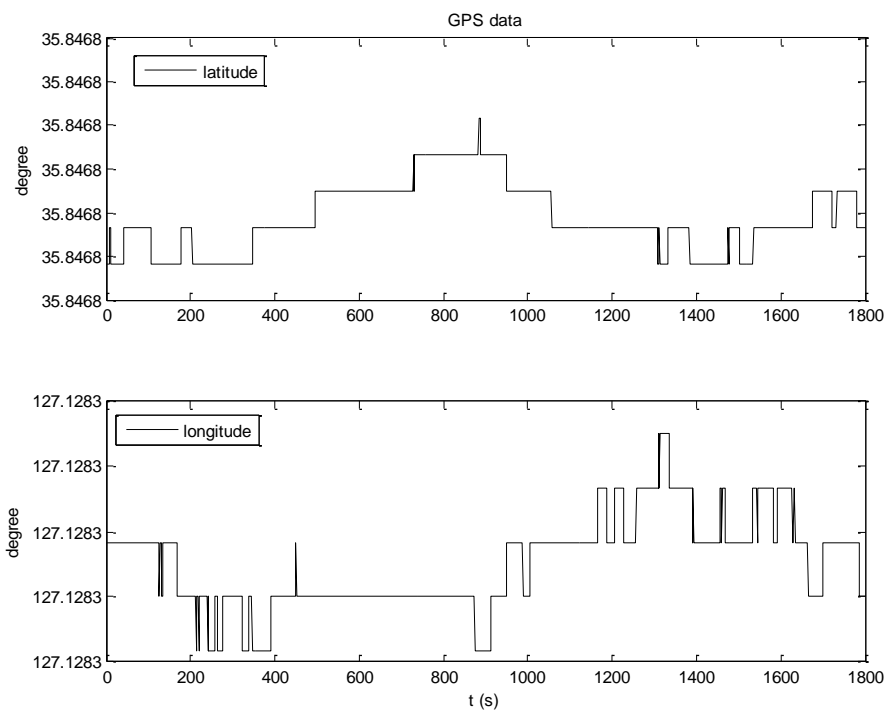

Figure 1 A Group of Typical GPS Data
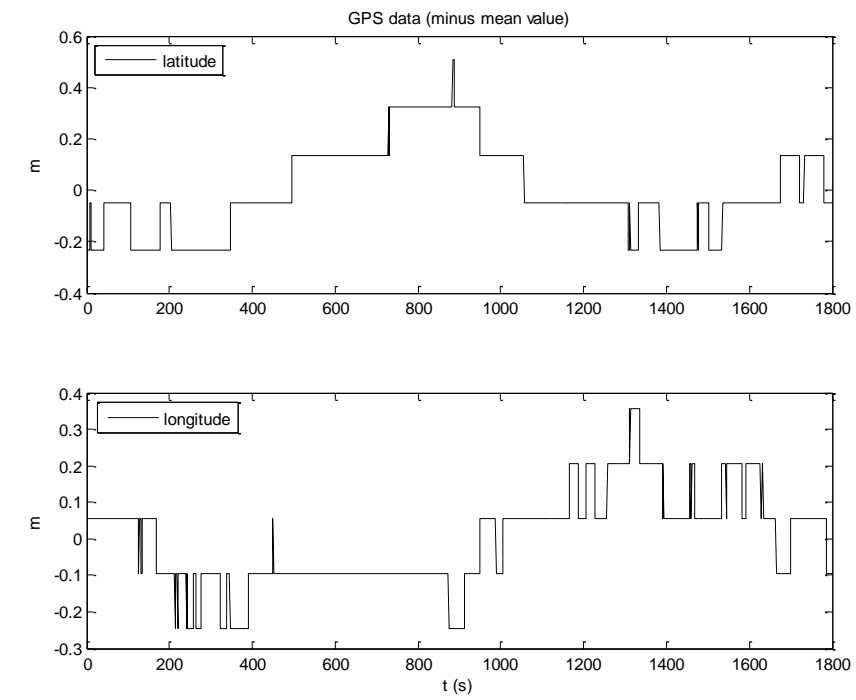

Figure 2. The Processed GPS Data After Subtracting the Mean Value 

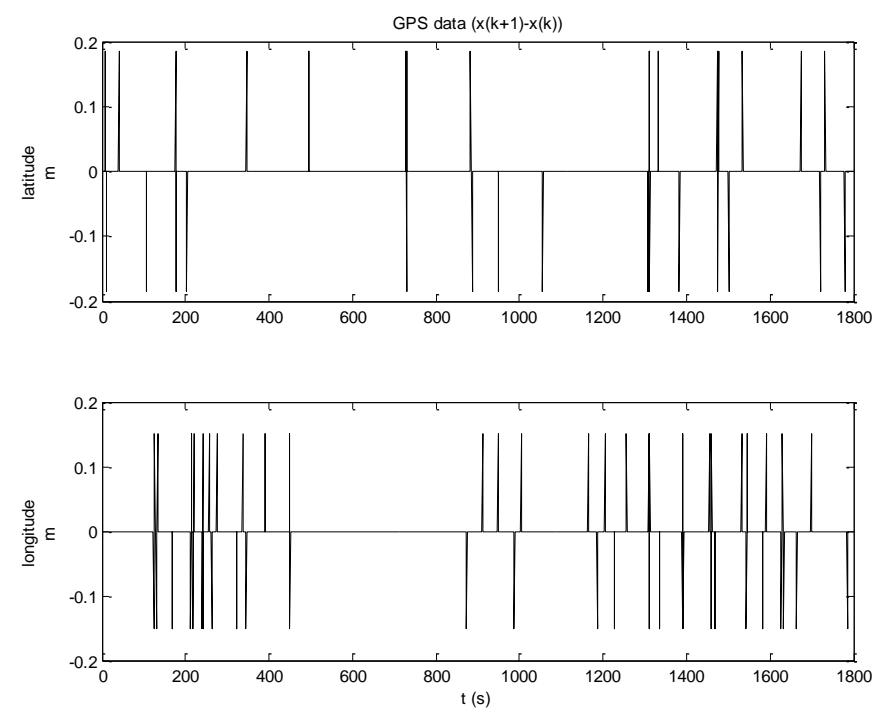

Figure 3. Difference Between the Adjacent Sampling Time GPS Data

\section{The Data Fusion Method}

In this paper a single GPS cooperated with DR is used to do the navigation for a mobile robot. DR is a very primitive positioning technique. For mobile robots traveling in a $2 \mathrm{D}$ planar space, it is possible to calculate the mobile robot position at any instance provided that starting location and all previous displacements are known. In this paper we use one electronic compass and two encoders located in the two driving wheels of the mobile robot to indicate the direction and velocity of the mobile robot. Fig. 4 presents a geometrical model of the wheeled mobile robot. This wheeled mobile robot has two driving wheels (radius $r$ ) and one caster. Point $H\left(x_{H}, y_{H}\right)$ defines the intersection of the axis of symmetry with the driving wheel axis, and is assumed to be the origin of the coordinate frame $\left\{X_{H}, Y_{H}\right\}$. Point $C\left(x_{c}, y_{c}\right)$ is the center of mass of the mobile robot. Length $c$ is the distance between point $H$ and point $C$, and $l$ is the length of the rear wheel axis. By knowing the direction $\theta$ and the velocity $v$, we can using Eq. (1) to calculate the position of the mobile robot.

$$
\left\{\begin{array}{l}
\dot{x}_{H}=v(t) \cos (\theta) \\
\dot{y}_{H}=v(t) \sin (\theta)
\end{array}\right.
$$

After obtaining the characteristics of the chosen GPS receiver's data the next step is to design the GPS/DR data fusion algorithm based on the obtained GPS characteristics. The short term error of DR is small but can accumulate over time without limitation. On the other hand the absolute error of GPS is big but with limitation. The errors of the chosen single GPS receiver are strongly colored. The characteristics of GPS data is that the errors change very slowly and every changing value is the resolution of $x$ and $y$ direction (for $x$ is $0.1854 \mathrm{~m}$ and for $y$ is $0.1503 \mathrm{~m}$ ). Here $x$ indicates latitude direction coordinate and $y$ indicates longitude direction coordinate. We assume that the reliability of the DR in $x$ and $y$ are $Q_{x}$ and $Q_{y}$, respectively; and the reliability of the 
chosen GPS receiver in $x$ and $y$ are $R_{x}$ and $R_{y}$, respectively. Of course $Q_{x}$ and $Q_{y}$ are much bigger than $R_{x}$ and $R_{y}$. The sketch of this data fusion process is shown in Fig. 5.

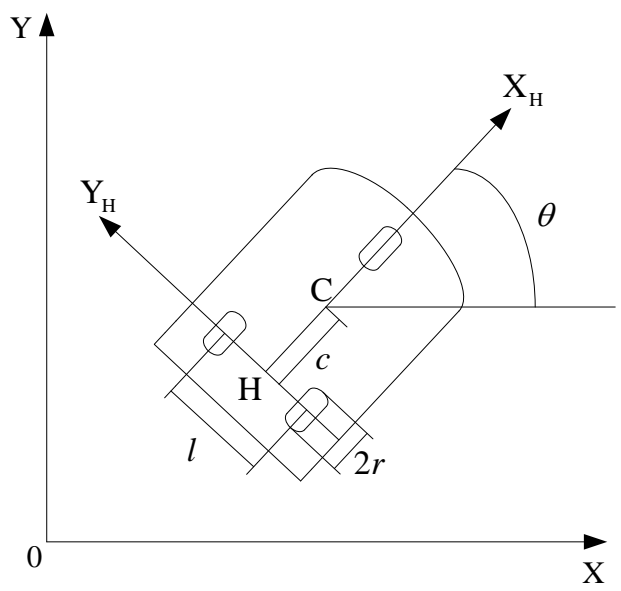

Figure 4. Sketch of a Three Wheeled Mobile Robot

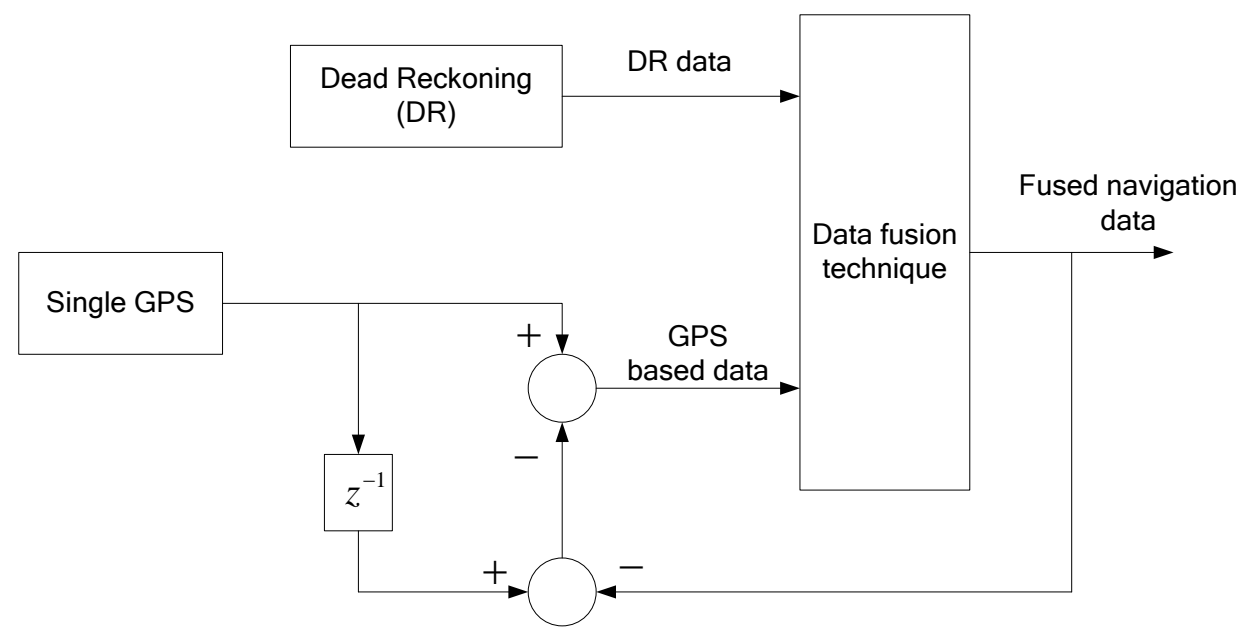

Figure 5. The Sketch of the Proposed Data Fusion Method

The details of the fusion steps are as follows. Considering the relative positioning problem, assume that there is an imaginary GPS receiver which has the same characteristics with the chosen single GPS receiver in the starting point.

1) At the starting point when the robot does not run collect the GPS data to get $G P S_{x}(0)$ and $G P S_{y}(0)$. Here $G P S_{x}(0)$ and $G P S_{y}(0)$ are the GPS output in the latitude and longitude direction at time $t=0 \mathrm{~s}$ in the start point, respectively. The imaginary GPS receiver which is fixed in the starting point has the same output of the chosen GPS receiver, i.e., $G P S_{I_{-} x}(0)=G P S_{x}(0)$ and $G P S_{I_{-} y}(0)=G P S_{y}(0)$. Here $G P S_{I_{-}}(0)$ and $G P S_{I_{-} y}(0)$ are the imaginary GPS receiver's output in the latitude and longitude direction at time $t=0 s$, respectively.

2) Run the robot. Since the frequency of the chosen single GPS receiver is $1 \mathrm{~Hz}$, the time interval used to do the navigation data fusion is $1 \mathrm{~s}$. At the time $t=1 \mathrm{~s}$ we can obtain 
the actual DR system based coordinate $x_{D R}(1)$ and $y_{D R}(1)$. Here $x_{D R}(1)$ and $y_{D R}(1)$ are the current position of the robot based on DR. Assume the reliabilities of $x_{D R}(1)$ and $y_{D R}(1)$ are $Q_{x}$ and $Q_{y}$, respectively.

3) At time $t=1 s$ we also get the actual GPS data: $G P S_{x}(1)$ and $G P S_{y}(1)$.

4) Now we consider the output of the imaginary GPS receiver fixed in the starting point. Since the errors of the chosen single GPS receiver change very slowly we can predict the current imaginary GPS receiver's output at time $t=1 \mathrm{~s}$ : $G P S_{I_{-} \hat{x}}(1)=G P S_{I_{-} x}(0)$ and $G P S_{I_{-} \hat{y}}(1)=G P S_{I_{-} y}(0)$. Here the "^" indicates that the output of the imaginary GPS receiver is the predicted data.

5) Then we can predict the robot's GPS based coordinate at time $t=1 \mathrm{~s}$ using the difference between the real and imaginary GPS receivers' output: $x_{G P S}(1)=G P S_{x}(1)-G P S_{I_{-} \hat{x}}(1)$ and $y_{G P S}(1)=G P S_{y}(1)-G P S_{I_{-} \hat{y}}(1)$. Here $x_{G P S}(1)$ and $y_{G P S}(1)$ are the current position of the robot based on GPS.

6) We set the reliabilities of $x_{G P S}(1)$ and $y_{G P S}(1)$, denoted by $R_{x}$ and $R_{y}$, respectively. Since DR can provide precise short term navigation information, we can use $x_{D R}(1)$ and $y_{D R}(1)$ to set the reliabilities of $x_{G P S}(1)$ and $y_{G P S}(1)$. For latitude direction, if $\left|x_{G P S}(1)-x_{D R}(1)\right| \leq 0.1854 n$ we set the reliability of $x_{G P S}(1)$ as $R_{x 1}$; If $\left|x_{G P S}(1)-x_{D R}(1)\right| \leq 0.1854 \times 2 m$ we set the reliability of $x_{G P S}(1)$ as $R_{x 2}$. Else, when $\left|x_{G P S}(1)-x_{D R}(1)\right| \geq 0.1854 \times 2 m$, the error of GPS jumps a lot. In this case GPS work in a weak situation so that the precision of GPS will degrade. We set the reliability of $x_{G P S}(1)$ as $R_{x 3}$. For longitude direction, if $\left|y_{G P S}(1)-y_{D R}(1)\right| \leq 0.1503 n$ we set the reliability of $y_{G P S}(1)$ as $R_{y 1}$; If $\left|y_{G P S}(1)-y_{D R}(1)\right| \leq 0.1503 \times 2 m$ we set the reliability of $y_{G P S}(1)$ as $R_{y 2}$. Else, if $\left|y_{G P S}(1)-y_{D R}(1)\right| \geq 0.1503 \times 2 m$ we set the reliability of $y_{G P S}$ (1) as $R_{y 3}$. Here some illustrations are given to explain the reliabilities of $x_{G P S}(1)$ and $y_{G P S}(1)$. Since DR's short term ( $1 s)$ precision is much better than that of the chosen single GPS receiver, $Q_{x}$ and $Q_{y}$ should be much larger than $R_{x i}$ and $R_{y i}(i=1,2,3)$, respectively. And the following inequalities exist.

$$
\begin{aligned}
& R_{x 1}>R_{x 2} \square R_{x 3} \\
& R_{y 1}>R_{y 2} \square \quad R_{y 3}
\end{aligned}
$$

7) Next, the fused results of DR and GPS based positioning results at time $t=1 \mathrm{~s}$ are calculated using the following equations.

$$
\begin{aligned}
& x(1)=\frac{Q_{x}}{Q_{x}+R_{x}} x_{D R}(1)+\frac{R_{x}}{Q_{x}+R_{x}} x_{G P S}(1) \\
& y(1)=\frac{Q_{y}}{Q_{y}+R_{y}} y_{D R}(1)+\frac{R_{y}}{Q_{y}+R_{y}} y_{G P S}(1)
\end{aligned}
$$

Here, $x(1)$ and $y(1)$ are fused robot's position results. The direction of the robot is measured using the electronic compass.

8) The current output of the imaginary GPS receiver fixed in the starting point is: 


$$
\begin{aligned}
& G P S_{I_{-} x}(1)=G P S_{x}(1)-x(1) \\
& G P S_{I_{-} y}(1)=G P S_{y}(1)-y(1)
\end{aligned}
$$

9) At time $t=k s(k=2,3 \ldots)$, we obtain the current time DR based positioning results: $x_{D R}(k)$ and $y_{D R}(k)$. The reliabilities of $x_{D R}(k)$ and $y_{D R}(k)$ are $Q_{x}$ and $Q_{y}$, respectively.

10) We then acquire the data from the real GPS: $G P S_{x}(k)$ and $G P S_{y}(k)$.

11) Predict the current time output of the imaginary GPS receiver: $G P S_{I_{-} \hat{x}}(k)=G P S_{I_{-} x}(k-1)$ and $G P S_{I_{-} \hat{y}}(k)=G P S_{I_{-} y}(k-1)$.

12) Predict the current time GPS based positioning results: $x_{G P S}(k)=G P S_{x}(k)-G P S_{I_{-} \hat{x}}(k)$ and $y_{G P S}(k)=G P S_{y}(k)-G P S_{I_{-} \hat{y}}(k)$.

13) Set the reliabilities of $x_{G P S}(k)$ and $y_{G P S}(k)$. This process is similar to Step 6.

14) Calculate the fused results of DR and GPS based positioning results at time $t=k s$ using the following equations.

$$
\begin{aligned}
& x(k)=\frac{Q_{x}}{Q_{x}+R_{x}} x_{D R}(k)+\frac{R_{x}}{Q_{x}+R_{x}} x_{G P S}(k) \\
& y(k)=\frac{Q_{y}}{Q_{y}+R_{y}} y_{D R}(k)+\frac{R_{y}}{Q_{y}+R_{y}} y_{G P S}(k)
\end{aligned}
$$

15) The current output of the imaginary GPS receiver fixed in the starting point is:

$$
\begin{aligned}
& G P S_{I_{-} x}(k)=G P S_{x}(k)-x(k) \\
& G P S_{I_{-} y}(k)=G P S_{y}(k)-y(k)
\end{aligned}
$$

\section{Simulation and Experiment}

\subsection{Simulation}

In this paper MATLAB is employed to do the data fusion simulation. We applied the proposed data fusion method and the Kalman filter technique, respectively. In the simulation the real GPS data is used. Totally 2200 seconds GPS data are collected. The fusion results obtained from the proposed data fusion method and the Kalman filter are compared with each other. Figures 6 and 7 show the fusion results. It can be seen that for the single GPS receiver the proposed data fusion method has better performance than the traditional Kalman filter. And in some special case, the GPS receiver may not work well. For example, it cannot detect enough satellites. In this case the error of GPS will be very large and the Kalman filter cannot provide good performance. Our proposed data fusion method can provide better performance by modifying the reliability of GPS. The reliability of GPS can be set very small if GPS is performing poorly and DR provides the navigaiton data in short term. Then recover the reliability of GPS when it works well again. 


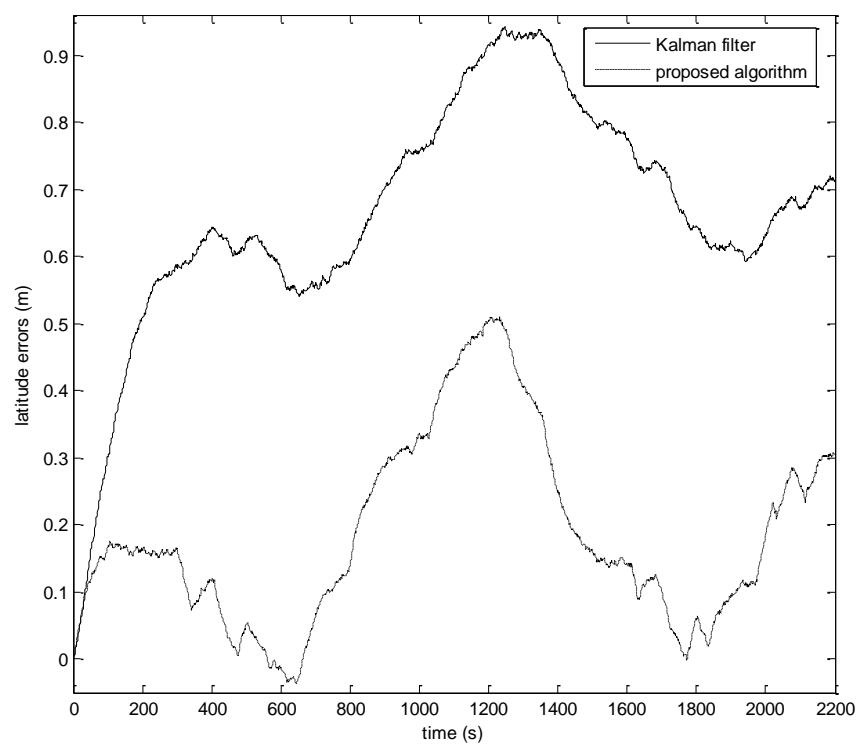

Figure 6. Latitude Errors of the Proposed Data Fusion Method and Kalman Filter

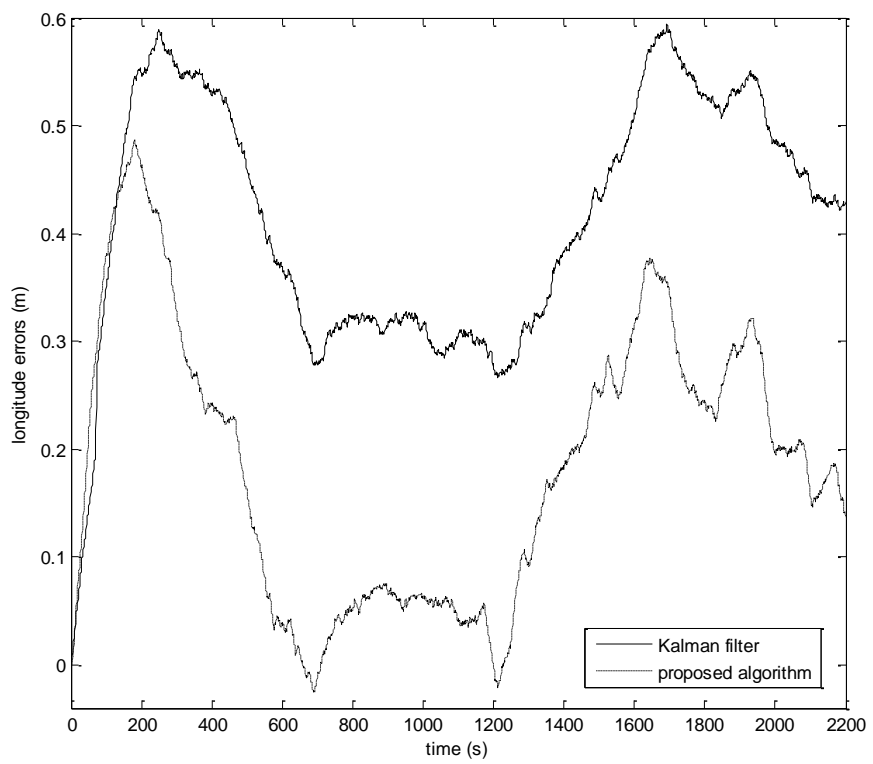

Figure 7. Longitude Errors of the Proposed Data Fusion Method and Kalman Filter

\subsection{Experiment}

The mobile robot 'DU ROBO' of our lab is used in the experiment. This robot has two driving wheels and one caster. Two encoders, one electrical compass and one single GPS receiver are set in the robot. The experiment is done in the playground of our 
school. In this experiment this robot runs a $10 \mathrm{~m} \times 10 \mathrm{~m}$ square three times. The route of the robot in the third circle is shown in Figure 8.

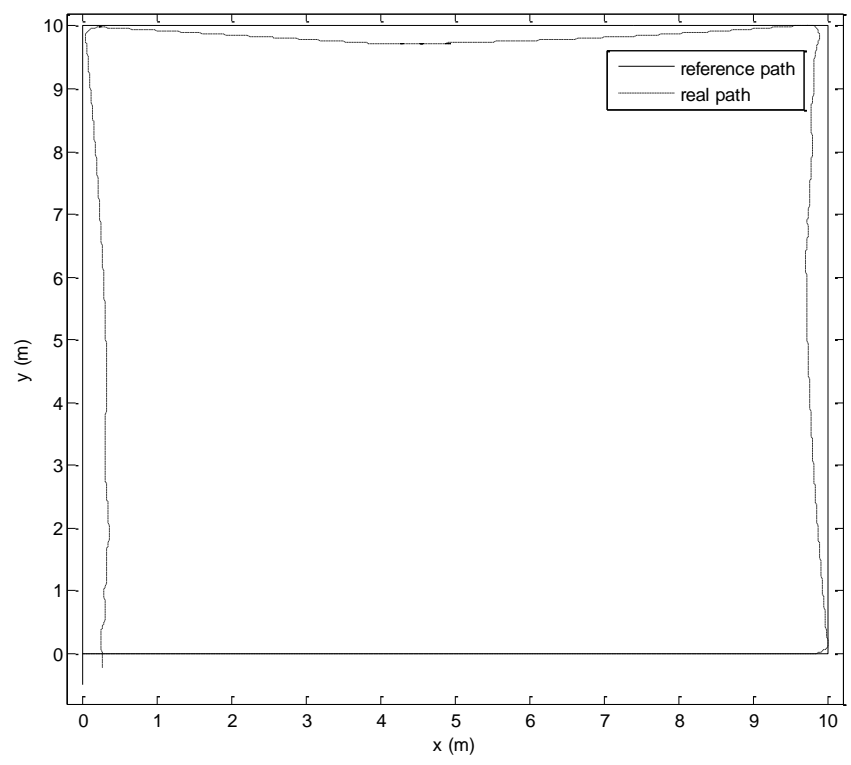

Figure 8. The Experiment Results

\section{Conclusion}

A precise and reliable navigation system is very important for the mobile robot application. For outdoor mobile robot GPS is usually used to do the navigation. In most of the cases DGPS is used because of its high precision. But the cost of DGPS is very high and DGPS needs a station to provide difference data. When the DGPS receiver cannot receive the difference data it will become a single GPS and its performance will degrade very much. The single GPS receiver's absolute errors are so big that it cannot be used to navigate the mobile robot alone. DR can provide short term precise navigation information but its errors can accumulate over time without limit. GPS has characteristics which are synergistic with DR. It can provide positioning information with a bounded error. In this paper a single GPS receiver is used to work in conjunction with DR. A new data fusion method which is designed basing on the characteristics of the single GPS receiver is proposed to fuse the data from DR and GPS. The proposed data fusion method can provide good fusion results. And when GPS's performance degrade due to the loss of the satellites the proposed data fusion method can also perform well by modifying the reliability of GPS. In this case only DR's data is used in short term. And when GPS recovers the proposed data fusion method can set back the reliability of GPS. Finally, the proposed data fusion method's computational burden is moderate. Simulation and experiment are conducted to verify this proposed data fusion method and the good results show the potential of this fusion method for the outdoor mobile robot navigation. 


\section{Acknowledgements}

This paper is supported by Jiangsu Province University Natural Science Research Project (13KJB510003) and also was financially supported by the Ministry of Trade, Industry \& Energy(MOTIE), Korea Institute for Advancement of Technology(KIAT) and Honam Institute for Regional Program Evaluation through the Leading Industry Development for Economic Region(R0001881), KRF (No. 2013068127).

\section{References}

[1] K. Hou, H. Sun, Q. Jia and Y. Zhang, "An autonomous positioning and navigation system for spherical mobile robot," Procedia Engineering, vol. 29, (2012), pp. 2556-2561.

[2] A. Noureldin, A. El-Shafie and M. Bayoumi, "GPS/INS integration utilizing dynamic neural networks for vehicular navigation," Information Fusion, vol. 12, no. 1, (2011), pp. 48-57.

[3] W. Seo, S. Hwang, J. Park and J.-M. Lee, "Precise outdoor localization with a GPS-INS integration system", Robotica, vol. 31, no. 3, (2013), pp. 371-379.

[4] R. Stancic and S. Graovac, "The integration of strap-down INS and GPS based on adaptive error damping," Robotics and Autonomous Systems, Vol. 58, (2010), pp. 1117-1129.

[5] S. Adusumilli, D. Bhatt and H. Wang, P. Bhattacharya and V. Devabhaktuni, "A low-cost INS/GPS integration methodology based on random forest regression," Expert Systems with Applications, vol. 40, (2013), pp. 4653-4659.

[6] D. Pazderski and P. Dutkiewicz, "Low-cost GPS Receivers in Navigation of Mobile Robots," Proceedings of Third International Workshop on Robot Motion and Control, (2002) November 1111, pp. 119-122, Bukowy Dworek, Poland.

[7] L. Jurišica, A. Vitko, F. Duchoň and D. Kaštan, "Statistical approach to GPS positioning of mobile robot," Journal of Control Engineering and Applied Informatics, vol. 12, no. 2, (2010), pp. 44-51.

[8] K. Lee, J. Park, O. Khatib and D.-S. Kwon, "Feedforward global/inertial sensor fusion algorithm for accurate global positioning of a mobile robot," Proceedings of the 2005 IEEE/ASME International Conference on Advanced Intelligent Mechatronics, (2005) July 24-28, pp. 504-509, Monterey, USA.

[9] J. Borenstein and L. Feng, "Measurement and Correction of Systematic Odometry Errors in Mobile Robots," IEEE Journal of Robotics and Automation, vol 12, no 6, (1996), pp. 869-880.

[10] A. Almagbile, J. Wang and W. Ding, "Evaluating the performances of adaptive Kalman filter methods in GPS/INS integration," Journal of Global Positioning Systems, vol. 9, no. 1, (2010), pp. 33-40.

[11] S. Han and J. Wang, "Integrated GPS/INS navigation system with dual-rate Kalman filter," GPS Solutions, vol. 16, no. 3, (2012), pp. 389-404.

[12] X. Chen, C. Shen, W.-B. Zhang, M. Tomizuka, Y. Xu and K. Chiu, "Novel hybrid of strong tracking Kalman filter and wavelet neural network for GPS/INS during GPS outages," Measurement, vol. 46, (2013), pp. 3847-3854.

[13] H. Zhang and Y. Zhao, "The performance comparison and analysis of extended Kalman filters for GPS/DR navigation,” Optik, vol. 122, (2011), pp. 777-781.

[14] S. Panzieri, F. Pascucci and G. Ulivi, "An outdoor navigation system using GPS and inertial platform," IEEE/ASME Transactions on Mechatronics, vol. 7, no. 2, (2002), pp. 134-142.

[15] S. Zeng, "Performance evaluation of automotive radars using carrier-phase differential GPS," IEEE Transactions on Instrumentation and Measurement, vol. 59, no. 10, (2010), pp. 2732-2741.

\section{Authors}

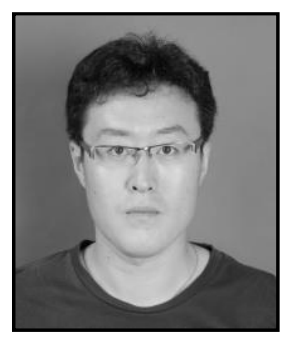

Yuanliang Zhang graduated from Tsinghua University, China in 2001. He received the M. Sc. and Ph.D. degrees from Chonbuk National University, Korea in 2006 and 2009, respectively. He is currently an associate professor at the School of Mechanical Engineering, Huaihai Institute of Technology, China. His research interests include control system and robotics. 


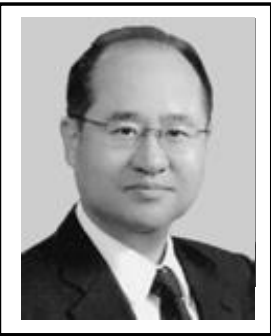

Kil To Chong (Corresponding author) received his Ph.D. in Mechanical Engineering from Texas A\&M University in 1995. Currently, he is a professor at the School of Electronics and Information Engineering at Chonbuk National University in Jeonju, Korea, and is head of the Advanced Research Center of Electronics. His research interests are in the areas of motor fault detection, network system control, time-delay systems, and neural networks. 
International Journal of Control and Automation Vol.7, No.10 (2014) 